\title{
DIAGNÓSTICOS DE CALIDAD PARA LA CONSERVACIÓN DE ESPACIOS AGRÍCOLAS ABANCALADOS. PROPUESTA METODOLÓGICA PARA LA CUENCA DEL GUINIGUADA (GRAN CANARIA, ISLAS CANARIAS)
}

\author{
L.E. Romero Martín, P. Ruiz Flaño y L. Hernández Calvento \\ Departamento de Geografía. Universidad de Las Palmas de Gran Canaria. \\ C/ Pérez del Toro, 1. 35003-Las Palmas de Gran Canaria \\ Lromero@dgeo.ulpgc.es, Pruiz@dgeo.ulpgc.es, Lcalvento@dgeo.ulpgc.es
}

\begin{abstract}
Resumen: En este trabajo se expone el método diseñado para el establecimiento de escalas de calidad para la conservación de un centenar de unidades de paisaje en las que domina la presencia de bancales. Dicho método tiene en cuenta las características productivas, ecológicas y paisajísticas de las áreas abancaladas. Los resultados obtenidos muestran que en la cuenca media y baja del Guiniguada (Gran Canaria), el espacio de bancales presenta una calidad para la conservación situada en niveles medios y altos. El 71\% de las unidades se sitúan en alguna de estas dos categorías, cifra elevada teniendo en cuenta que el $60 \%$ de las mismas está abandonada.
\end{abstract}

Palabras clave: Terrazas agrícolas, unidades de bancales, calidad para la conservación, diagnóstico ambiental, Islas Canarias

\begin{abstract}
In the present text it's exposed the designed method to set up the ranges of quality for the conservation of a hundred of unit of landscape where the presence of terraces is dominant. This method value the characteristics of those unit relative of it's worth in productivity, ecology and scenery. The obtained results show that in the half and low areas of the Guiniguada valley, the spaces occupied by terraces present between a medium and high levels of quality for the conservation. $71 \%$ of the units are qualified by someone of these two categories, a high number bearing in mind that $60 \%$ of them are abandoned.
\end{abstract}

Key words: Agricultural terraces, units of terraces, quality for the conservation, environmental diagnostic, Canary Islands 


\section{Introducción}

Los bancales son el resultado de la fuerte transformación a que se han visto sometidas numerosas vertientes con el fin de incrementar la superficie agrícola en lugares donde ésta era escasa. Las laderas son transformadas y convertidas en un escalonamiento de rellanos de cultivo, con sus respectivos saltos, cuyas dimensiones, formas y alturas varían notablemente en función de la pendiente y de la forma de la vertiente.

Las ventajas que derivan del aterrazamiento justifican, en parte, el esfuerzo humano empleado en su creación. Estas superficies llanas favorecen la infiltración del agua de lluvia, influyen en el incremento de la productividad y de la potencia edáfica, constituyen una medida eficaz contra la erosión y, en aquellos bancales que disponen de muros de piedras, éstos ejercen de reserva térmica por el calor acumulado en su masa, lo que favorece la existencia de determinados cultivos (Ambroise $e t a l$., 1989). Son, además, una manifestación de ingeniería popular, por lo que constituyen un importante valor patrimonial, producto de una cultura histórica de la piedra, al tiempo que conforman, en la mayor parte de los casos, paisajes de gran belleza y valor visual (Colomar Marí, 2002).

La bibliografía científica se ha ocupado de ellos de forma importante, centrándose fundamentalmente en su origen geográfico (Spencer y Hale, 1961; Wheatley, 1965), en su tipología (Lasanta, 1989 y 1990; Pérez Cueva, 1985), en los problemas de evolución ecológica tras su abandono (García Ruiz et al., 1988; Rodríguez Aizpeolea, 1990; Llorens et al., 1992), etc. Rodríguez Aizpeolea y Lasanta (1992) y el monográfico 71 (3-4) de Méditerranée realizan excelentes síntesis de los trabajos sobre bancales realizados hasta ese momento. Sin embargo, se ha descuidado su tratamiento como patrimonio a conservar. No puede olvidarse que constituyen paisajes de gran riqueza desde el punto de vista estético, arquitectónico, etnográfico y edáfico, y que tras su abandono comienzan a mostrar su apariencia más desoladora.

Es precisamente la degradación de las funciones ecológicas y visuales de estos espacios lo que ha motivado el interés de organizaciones, nacionales e internacionales, por patrocinar o promover estudios relacionados con su protección y mantenimiento y con sus futuras posibilidades de explotación. En el ámbito europeo, el Programa Terrazas (1981-1989), iniciado por la Asociación APARE (Association pour la Participation et la Action Regionale), es el pionero en este tipo de iniciativas. Con él se promovieron trabajos de rehabilitación de terrazas de cultivo y programas experimentales sobre nuevos usos agrícolas, turísticos y pedagógicos para los bancales de la zona mediterránea francesa. A este programa siguieron otros, financiados por la Unión Europea, como el Programa ProTerra (1996-2001), que integró 14 proyectos desarrollados en los países europeos de la orla mediterránea, con el objetivo de desarrollar un programa experimental de revalorización de sus espacios abancalados. 
Con carácter más reciente se ha desarrollado el Proyecto Patter (1999-2001), en el que participan el Consell de Mallorca, y las Universidades de Niza y Génova, cuyo objetivo es la sistematización de las tareas de catalogación, análisis y diagnóstico de los territorios abancalados.

En este sentido, el trabajo que aquí se presenta comparte objetivos con los anteriores. En él se plantea el diseño metodológico y los resultados obtenidos en un análisis de calidad para la conservación de los espacios aterrazados de la cuenca del Guiniguada, en Gran Canaria. Este trabajo nace del encargo realizado por el Cabildo de Gran Canaria a miembros del Grupo de Investigación de Geografía Física y Medio Ambiente de la Universidad de Las Palmas de Gran Canaria, en el sentido de proponer actuaciones para conservar y restaurar las terrazas agrícolas de un sector de esta cuenca. Como paso previo a la restauración, se hizo necesario el diseño de una metodología que permitiese establecer categorías de calidad para la conservación. Basándose en estas categorías, y teniendo en cuenta la viabilidad, se procedería a la restauración de los bancales de mayor calidad.

\section{2. Área de estudio}

Este estudio se centra en los tramos medio y bajo de la cuenca del Guiniguada, y más concretamente en el espacio comprendido entre el núcleo de Santa Brígida y El Pambaso. Se localiza en el sector nororiental de la isla de Gran Canaria (Figura 1) y cuenta con una superficie de $14,53 \mathrm{Km}^{2}$, lo que supone el $22,3 \%$ del espacio total de dicha cuenca.

La variedad litológica es una de sus características dominantes, pues coexisten materiales volcánicos antiguos (fonolitas e ignimbritas del primer ciclo) con depósitos y edificios del volcanismo reciente y otros de origen sedimentario, de diversas edades y orígenes. De forma general, el relieve asciende de NE a SE, correspondiendo al Pico de La Atalaya y al Llano de la Cruz, con $706 \mathrm{~m}$ y $655 \mathrm{~m}$ de altitud respectivamente, los puntos más elevados, situados en el sector suroriental.

La dirección dominante de la red de drenaje es la NE-SW y su trazado es lineal, a excepción del tramo final, donde el cauce principal se torna meandriforme.

La alternancia entre procesos erosivo-acumulativos y erupciones volcánicas sobre una misma línea de drenaje ha producido un relieve con pendientes moderadas donde alternan barrancos encajados e interfluvios alomados, a los que se superpone un importante número de edificios volcánicos pertenecientes a los periodos constructivos más recientes. 

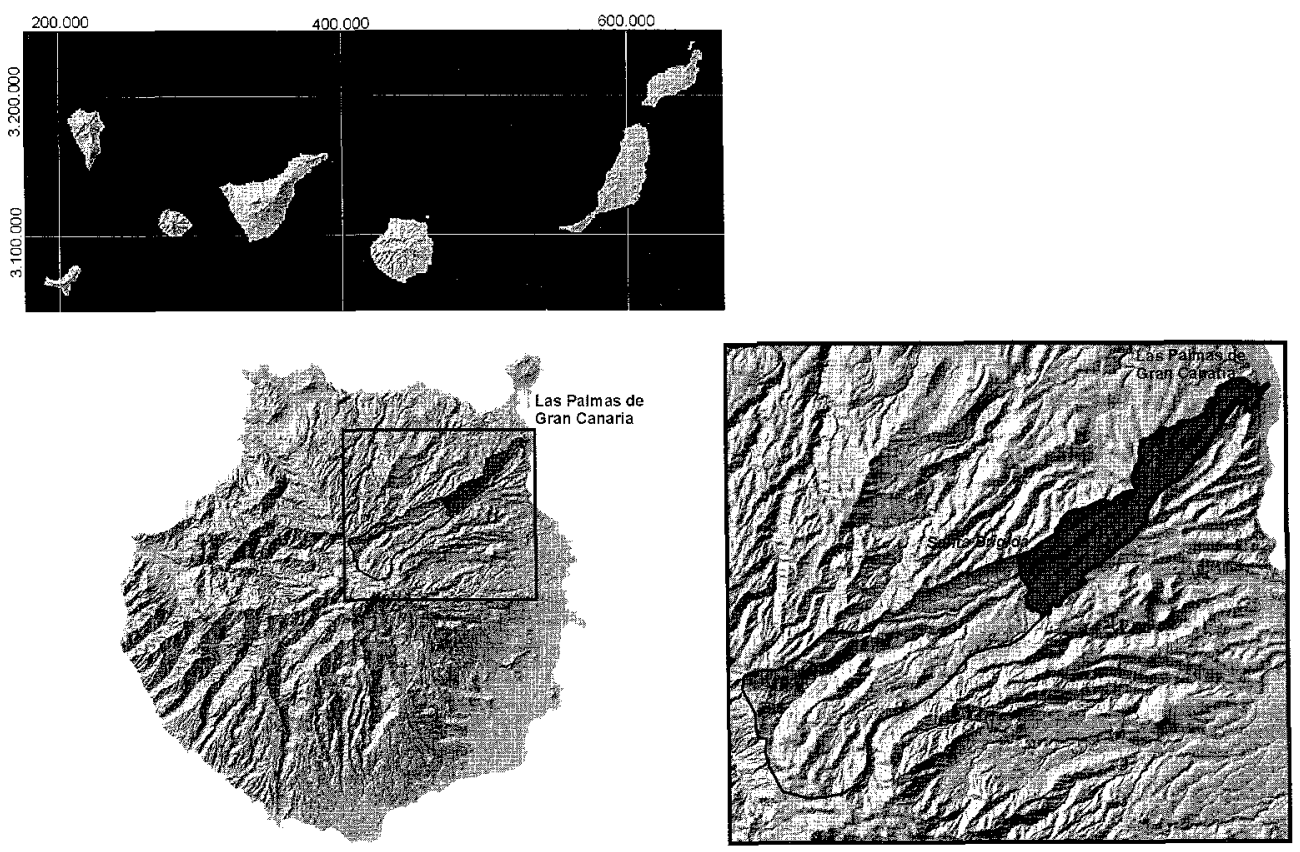

Figura 1. Cuenca del Guiniguada. Gran Canaria. Islas Canarias.

Sus características climáticas son las propias de los sectores de barlovento de las Islas Canarias. El área se encuentra regularmente afectada por los vientos alisios, cuya interferencia con el relieve origina el escalonamiento del régimen termopluviométrico, así como la existencia de una amplia gama de microclimas (Marzol, 1987). La temperatura media del aire oscila entre $20,9^{\circ} \mathrm{C}$ de Las Palmas de Gran Canaria, ciudad situada a nivel del mar, y $15,4^{\circ} \mathrm{C}$ de Tafira, a 337 metros de altitud. Los valores de lluvia anual oscilan entre $208 \mathrm{~mm}$ en la costa (Llano de Las Brujas) y $413 \mathrm{~mm}$ en Santa Brigida, en el interior y a 475 metros de altitud aproximadamente (Romero y Hernández, 1996).

El paisaje vegetal se encuentra sumamente transformado florística, fisionómica y espacialmente. La desaparición de las formaciones arbóreas originales ha dado paso al dominio del estrato arbustivo, donde conviven especies propias del matorral degradado de cada piso bioclimático con especies transgresivas de los pisos contiguos, junto con otras introducidas para su cultivo, hoy asilvestradas.

Por lo que respecta a las características socioeconómicas, se trata de un espacio sumamente antropizado, en el que la ocupación humana ha seguido un ritmo cre- 
ciente hasta la actualidad, momento en el que la densidad de población es muy elevada $\left(1412\right.$ personas $\left./ \mathrm{km}^{2}\right)$.

El Guiniguada medio y bajo forma parte del área metropolitana o de expansión de Las Palmas de Gran Canaria, por lo que sus núcleos de población se han convertido en barrios dormitorios de la población activa, que es atraída por la capital insular. Desde el punto de vista territorial se pone de manifiesto la dura competencia por la ocupación del suelo entre el uso urbano y el agrícola. El cese de la actividad agraria se evidencia en el aumento del $26,3 \%$ de la superficie agricola abandonada en el intervalo 1960-1992, y en la recolonización vegetal, cobertura que aumenta en un $5,2 \%$ en el mismo periodo.

Por último, y como prueba de la amplia tradición agrícola que caracteriza este espacio, se encuentra el hecho de que algo más de mitad de su superficie ha sido cultivada en alguna ocasión y al menos el $50 \%$ de ésta última se ha explotado mediante el sistema de terrazas.

\section{Metodología}

Ante la imposibilidad de trabajar los bancales como unidades independientes, se adoptó la decisión de agruparlos en unidades de características ecoantrópicas semejantes, que sustituyen a la unidad homogénea de paisaje habitualmente empleada por los estudios integrados de paisaje (Bovet y Ribas, 1992). De acuerdo con los procedimientos utilizados en estos mismos estudios, la metodología continúa con la realización del inventario y con el establecimiento del diagnóstico ambiental. A partir del inventario de las unidades de bancales se crea una base de datos y se procede a la determinación de las categorias de calidad para la conservación.

El diagnóstico final de calidad para la conservación se concibe como una expresión sintética del valor del territorio (Tavío et al., 2002), en este caso del valor de unidades de bancales. Por ello debe agrupar e integrar todos aquellos aspectos susceptibles de justificar la protección y conservación de los mismos, aspectos que en nuestro estudio han sido los siguientes: valor productivo, ecológico y estético o visual. Para su obtención se elabora un algoritmo en el que están representados estos valores mediante diferentes parámetros. Y así, la calidad del suelo se selecciona como parámetro representativo del valor productivo; el papel regulador de la dinámica hidrológica de la vertiente lo es del valor ecológico, mientras las características constructivas, la calidad visual y la integración de las actividades humanas en la unidad de bancales lo son del valor estético. La obtención de cada uno de estos parámetros requiere valoraciones específicas que se presentan a continuación. 
a) Calidad del suelo (CS). Su consideración se justifica por la necesidad de preservar los escasos suelos agrícolas de calidad que existen en la isla. En el caso de los bancales, el trabajo realizado por los agricultores a lo largo de decenas de años ha permitido la acumulación y evolución de suelos productivos, cuya conservación es imprescindible, de dónde deriva el elevado peso de este parámetro dentro del algoritmo final. Para su determinación se retoman las clases de capacidad de uso agrario establecidas por Sánchez et al. (1995), ya que integran características y limitaciones del suelo, agrupándolas en tres valores. El mayor valor (valor 3) es el que alcanzan los suelos de la clase B, de elevada capacidad de uso. Le siguen los suelos de la clase C (valor 2), con capacidad de uso moderada. Finalmente, los suelos de baja calidad (valor 1) integran todos los de la clase D y E, de baja capacidad de uso.

b) Papel regulador de la unidad de bancales en la dinámica hidrológica de la vertiente (PR). Con este parámetro se reconoce el papel ecológico que desempeñan los bancales dentro de las laderas. Su capacidad de retención de agua, potenciando la infiltración, y la consiguiente reducción de la escorrentía superficial, evitan el desarrollo de procesos de erosión de magnitud que pudieran originar una degradación importante de las vertientes. Se han distinguido tres tipos de regulación (alta, media y baja), obtenidos a partir del grado de ocupación de la vertiente por parte de los bancales y el estado de conservación de los mismos. El valor mínimo se reserva a aquellas situaciones en que la unidad de bancales ocupa sectores localizados de la ladera o bien tiene muros deteriorados, mientras que el valor más alto se otorga a los bancales de rellano plano, bien conservados, y que ocupan una importante superficie.

c) Caracteristicas constructivas de los muros (CC). Varias son las causas de su inclusión y su notable peso dentro del algoritmo final. Señalamos entre ellas la elevada inversión de capital humano que requirió su construcción, la apreciable calidad estética de algunos muros y el hecho de que estas características intervienen de forma decisiva en el mantenimiento y conservación de las estructuras a largo plazo. Su valor final viene establecido por el sumatorio de los valores parciales obtenidos de la valoración de dos aspectos: tipo de mampostería y materiales empleados en la construcción.

La mampostería es un reflejo de la solidez de los muros y de su resistencia a procesos como desprendimientos y deslizamientos En este sentido, se otorgan valores más elevados a los muros construidos mediante hileras (valor 3), que a los elaborados mediante mampostería ordinaria y de rajuela (valor 2) y a la de cantos rodados (valor 1). Por su parte, los materiales se valoran en función de su rareza y compresividad, de acuerdo a una escala de 4 a 1 , en la que las ignimbritas alcanzan el valor más elevado, seguidas de fonolitas, cantos rodados y, en último lugar, basaltos.

d) Calidad visual de la unidad de bancales (CV). Pese a la carga de subjetividad que encierra, se ha tomado en consideración este parámetro al comprobar que las cualidades estéticas de algunas unidades resaltan su valor para la conservación. Es 
además el de obtención más compleja ya que requiere la consideración de una serie de valores parciales, oportunamente ponderados, referidos a diferentes características o cualidades presentes tanto en la unidad de bancales como en su entorno. Estas características son el contraste cromático de la vegetación dentro de la unidad, valor de la vegetación del entorno, existencia de relieves singulares, existencia de afloramientos rocosos y fondo escénico.

El contraste cromático de la vegetación dentro de la unidad refleja la incidencia del colorido de la vegetación en su interior, obteniendo la puntuación más alta las unidades abandonadas que hayan alcanzado un mayor grado de diversidad vegetal en el proceso recolonizador. También se ha aplicado a los campos cultivados, pero, salvo en contadas ocasiones, se trata de cultivos monoespecíficos.

Para determinar el valor de la vegetación del entorno se tienen en cuenta tanto su singularidad y densidad como su contraste cromático. Ambos aspectos, además de realzar la calidad visual de la unidad, consideran la existencia de especies que cuentan con algún grado de protección según la Orden de 20 de febrero de 1991, sobre Protección de Especies de la Flora Vascular Silvestre de la Comunidad Autónoma de Canarias. Los valores más elevados de este parámetro corresponderán a aquellas unidades en cuyo entorno existe una alta densidad de elementos vegetales singulares y un gran contraste cromático. En el extremo inferior se sitúan las unidades sin elementos singulares en el entorno y de bajo contraste cromático.

La existencia de relieves singulares contempla la presencia o ausencia de aspectos geomorfológicos tales como escarpes, meandros en el fondo del barranco, o conos volcánicos, que pueden contribuir a incrementar la calidad visual del paisaje.

Con la existencia de afloramientos rocosos se valora la presencia y densidad de los mismos, al considerar, como así se ha percibido en el campo, que estos realzan su calidad visual. Los valores más elevados (valor 3) se asignan a las unidades donde los afloramientos son abundantes y los menores (valor 1) donde existen pero son poco frecuentes.

Por lo que respecta al fondo escénico, se ha considerado que la calidad visual de las unidades de bancales no depende exclusivamente de ellas, sino también de su entorno, el cual puede contribuir a realzar, en distinta medida, los valores estéticos del conjunto. Por ello se ha otorgado un valor especial (valor 5) a aquellas unidades cuya calidad visual se ve potenciada de forma notable por el paisaje circundante.

e) Integración de las actuaciones humanas. Con él se han tenido en cuenta las actuaciones humanas dentro de la unidad de bancales como un parámetro que eleva la calidad de aquellas unidades en las que existen valores a conservar, como construcciones o edificaciones tradicionales relacionadas con el uso agrícola o agrario (alpen- 
dres, cuartos de aperos, estanques, maretas, minas, acequias, casas, etc.). Las puntuaciones más elevadas corresponden a los bancales en los que las actuaciones inciden favorablemente sobre la unidad o bien a aquellos en los que no existe dicha actuación. Sin embargo, no se penaliza a las unidades con actuaciones de carácter negativo (basuras, casetas de baja calidad constructiva...) pues éstas pueden desaparecer con una tarea de limpieza y, por tanto, deben intervenir poco en la calidad para la conservación final.

Elaborados los valores parciales de diagnóstico, éstos se sintetizan en un último valor, el de calidad para la conservación, que queda expresado en el siguiente algoritmo:

$$
5 \mathrm{CS}+5 \mathrm{PR}+3 \mathrm{CC}+\mathrm{CV}+\mathrm{IAH}
$$

donde $\mathbf{C S}=$ calidad del suelo; $\mathbf{P R}=$ papel regulador en la dinámica hidrológica de la vertiente; $\mathbf{C C}=$ características constructivas $\mathbf{C V}=$ calidad visual y $\mathbf{I A H}=$ integración de las actuaciones humanas.

La aplicación de este algoritmo permitió el establecimiento de tres categorías de calidad para la conservación (baja, media y alta), en las que quedan incluidas todas las unidades de bancales del tramo inferior de la cuenca del Guiniguada.

\section{Resultados}

El trabajo de fotointerpretación y de campo permitió la identificación de 111 unidades de bancales en los tramos medio e inferior de la cuenca del Guiniguada. Lejos de conformar espacios homogéneos, se caracterizan por la gran variedad de situaciones ambientales y antrópicas que presentan. Así, en referencia a sus características ambientales, cabe señalar, a modo de resumen, su localización preferente en exposiciones norte ( $45 \%$ de los casos), en laderas cóncavas y partes inferiores de las vertientes y en pendientes comprendidas entre $15^{\circ}$ y $20^{\circ}$. Por lo que respecta a los parámetros antrópicos, la situación de abandono es la dominante (60\% de las unidades) y, en la mayor parte de los casos, se trata de abandonos posteriores a 1996.

Tabla 1. Diagnóstico final de calidad para la conservación. Fuente: Elaboración propia.

\begin{tabular}{|ccccc|}
\hline $\begin{array}{c}\text { Calidad para } \\
\text { la conservación }\end{array}$ & $\begin{array}{c}\text { Unidades } \\
\mathrm{N}^{\circ}\end{array}$ & $\begin{array}{c}\text { Unidades } \\
\%\end{array}$ & $\begin{array}{c}\text { Superficie } \\
\text { Has }\end{array}$ & $\begin{array}{c}\text { Superficie } \\
\%\end{array}$ \\
\hline Baja & 32 & 28,8 & 40,4 & 20,8 \\
Media & 53 & 47,7 & 96,9 & 50,0 \\
Alta & 26 & 23,4 & 56,7 & 29,2 \\
\hline
\end{tabular}




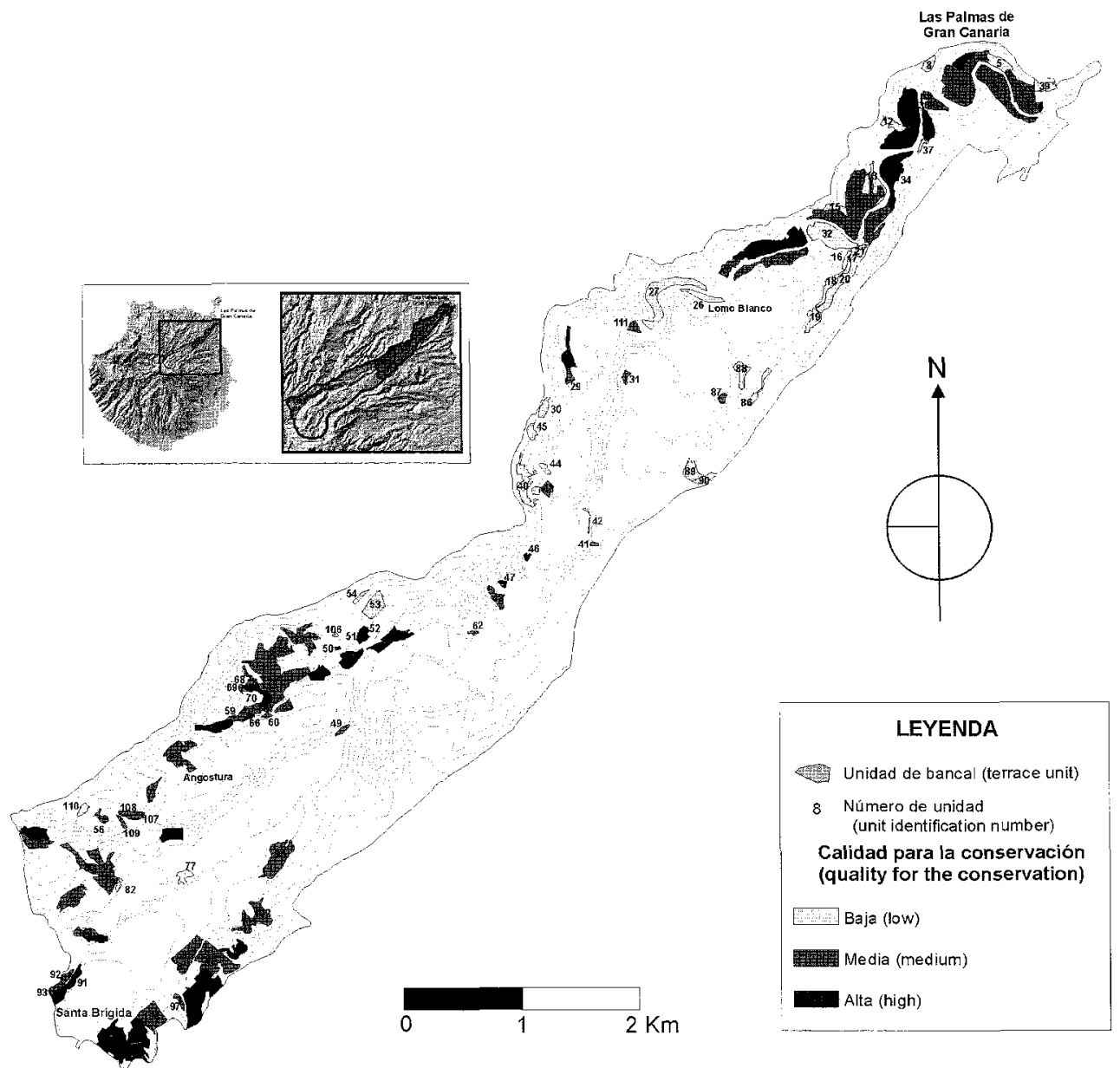

Figura 2. Calidad para la conservación de las unidades de Bancales. Cuenca del Guiniguada.

La tabla 1 refleja los resultados de calidad para la conservación obtenidos tras la aplicación del algoritmo. La calidad media para la conservación es la categoría mejor representada, pues aproximadamente la mitad de las unidades entran en ella.

Las unidades con baja calidad para la conservación son 32. Esta categoría es frecuente en los sectores marginales del tramo bajo del Barranco Guiniguada y está especialmente concentrada en el Barranquillo de los Toledo (Figura 2). La figura 3 pone de manifiesto la frecuencia de los distintos parámetros de diagnóstico de estas unidades. En ellas predominan los suelos de baja y media capacidad de uso agrario. Son fre- 


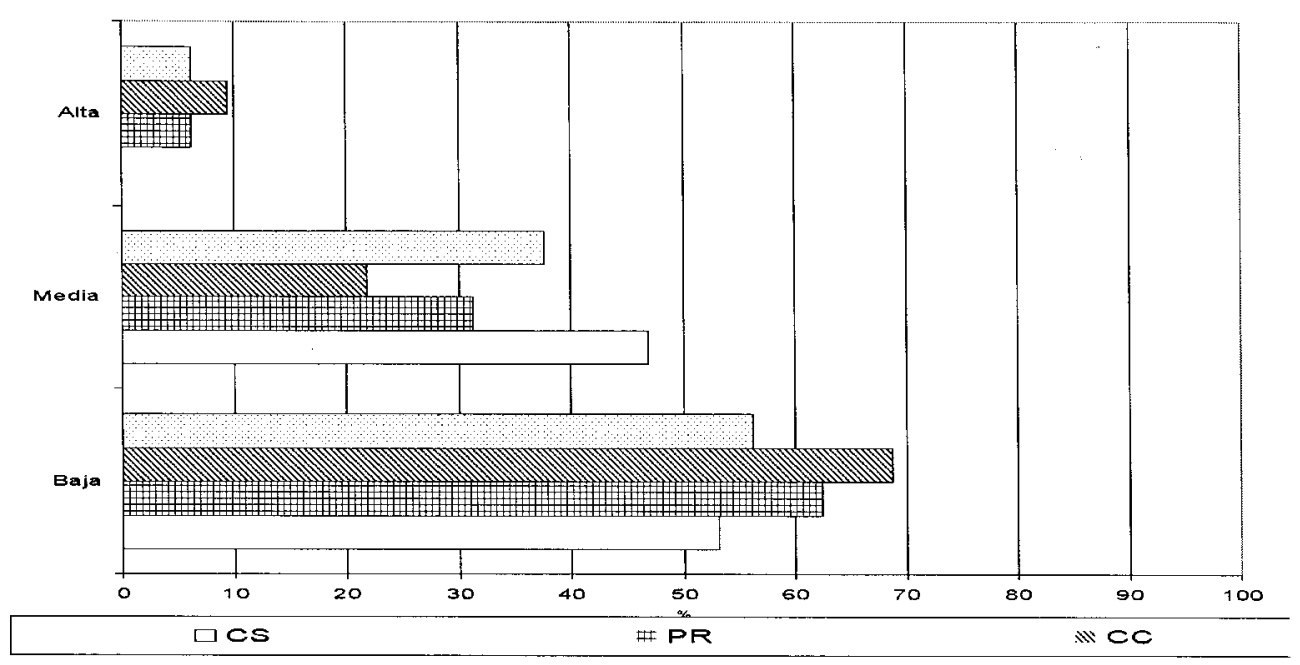

Figura 3. Frecuencia de los parámetros CS, PR, CC y CV en unidades con calidad para la conservación baja. Fuente: Elaboración propia.

cuentes los muros de contención de mampostería de cantos rodados y de mampostería ordinaria, tipologías constructivas donde las piedras, tanto por su morfología como por su disposición irregular, hacen de los muros paramentos poco estables:

La mayor parte de estas unidades presenta una limitada capacidad de captación de agua de escorrentía debido a su reducida ocupación superficial, pues suelen ser unidades con un escaso número de bancales, con sus rellanos en pendiente y alto grado de deterioro.

Quizás lo más destacable de estas unidades sea su reducido valor paisajístico. Dominan los bancales abandonados con incipiente y pobre recolonización vegetal y con infraestructuras agrícolas deterioradas o de baja calidad constructiva, lo que provoca un fuerte impacto visual (cortavientos de bidones, invernaderos, casetas de bloques sin encalar, etc). Además suelen situarse en vertientes con escaso recubrimiento vegetal y con formaciones de matorral degradado de carácter xerofítico, en el sector bajo de la cuenca, como tabaibas (Eupborbia regis-jubae), tuneras (Opuntia ficusbarbarica), pitas (Agave americana), aulagas (Launaea arborescens), etc; y de carácter termófilo en el sector medio, como vinagreras (Rumex lunaria), granadillo (Hypericum canariense), etc.. La mayoría de ellas se encuentra enmarcada por espacios urbanos de escaso valor arquitectónico y con fuertes impactos ambientales (pésima calidad constructiva, basuras, escombros, torreones de luz, taludes artificiales, puente de la circunvalación, etc.). 


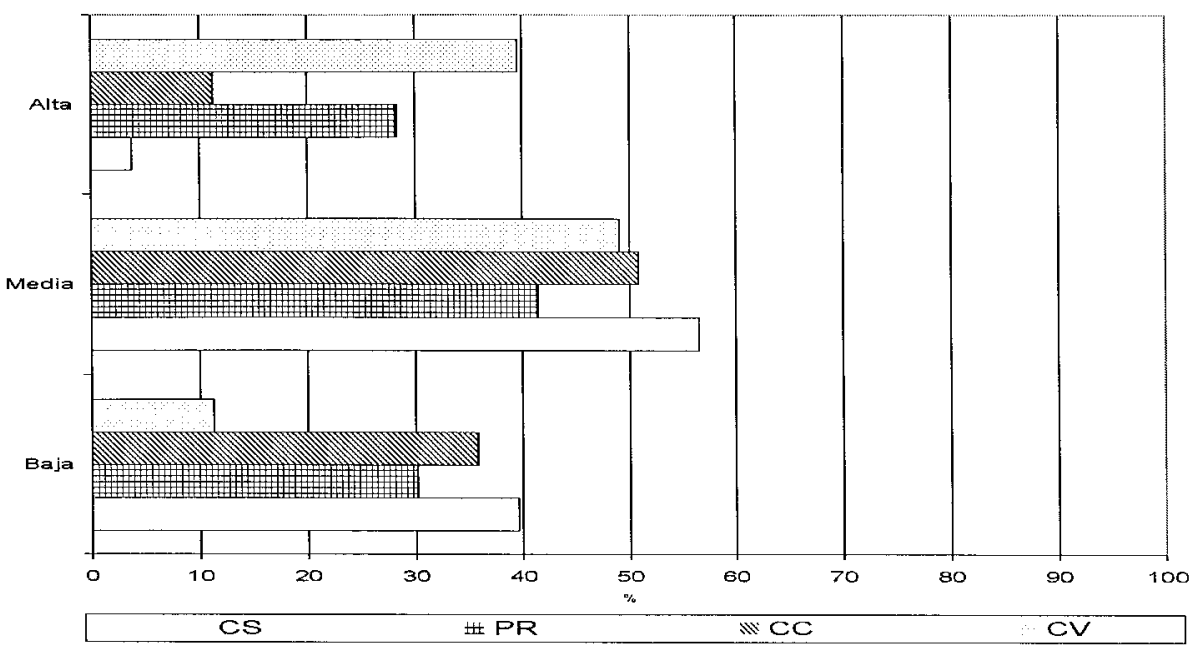

Figura 4. Frecuencia de los parámetros CS, PR, CC y CV en unidades con calidad para la conservación media. Fuente: Elaboración propia.

Las unidades con moderada calidad para la conservación son las más abundantes. Constituyen un grupo de 53 unidades que se distribuyen por toda la superficie de la cuenca baja y media del Guiniguada. Se caracterizan por presentar valores medios de los parámetros productivos, hidrogeomorfológicos y paisajísticos (Figura 4). Alrededor del $60 \%$ de estas unidades pertenece a la clase agrológica $\mathrm{C}$, es decir, presentan un valor medio de capacidad de uso agrario. Le siguen en importancia los suelos con capacidad de uso baja (clases D y E), con un porcentaje de frecuencia del 34\%.

Por lo que respecta a su valor hidrológico, se trata de un grupo sumamente heterogéneo, con un peso muy similar de las categorías bajo, medio y alto papel regulador de la dinámica. Algo similar ocurre con las características constructivas de los muros. El 50\% está construido con mampostería de calidad media (ordinaria y rajuela), seguidos por de calidad baja (mampostería de cantos rodados) con el 35,8\% y el resto $(11 \%)$ se ha construido con mampuestos regulares por hileras.

Por último, este grupo presenta un claro dominio de los valores de calidad visual o paisajística media-alta, pero, al hallarse el resto de los parámetros de la valoración muy distribuidos entre las distintas categorías, no han podido incluirse en el grupo de calidad para la conservación alta.

El número de unidades con alta calidad para la conservación asciende a 26 y, aunque están presentes en todos los sectores de la cuenca baja y media del Guiniguada, 


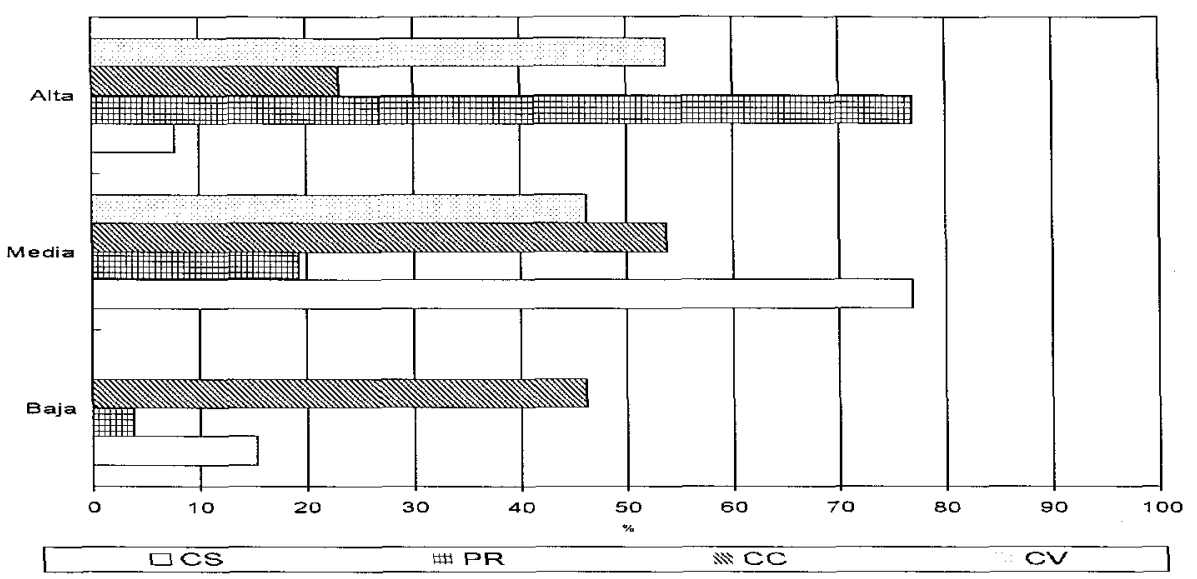

Figura 5. Frecuencia de los parámetros CS, PR, CC y CV en unidades con calidad para la conservación alta. Fuente: Elaboración propia.

podemos destacar tres enclaves importantes: Meandros de la cuenca baja, Palma de Siete Puertas y Andújar y cabecera del Barranco del Colegio. Las características más sobresalientes de este grupo se centran en su importante papel regulador de la hidrología de la vertiente y su elevada calidad visual o paisajística (Figura 5).

En ellas predominan los suelos con capacidad de uso agrario medio, es decir, los de la clase $\mathrm{C}$. Cabe destacar que dentro de este grupo se localizan dos de las cuatro unidades encontradas en los bancales del Guiniguada con capacidad de uso agrario alto (clase B).

La tipología constructiva de los muros de contención y la piedra empleada en la obra son muy variados y, aunque predomina la mampostería de calidad constructiva media (mampostería ordinaria), con la piedra más común (el basalto), existen bellos ejemplares de mampostería de rajuela de fonolitas y de mampostería en hileras de ignimbritas, consideradas estas últimas por su óptima calidad constructiva.

Dominan los bancales con rellanos planos, que ocupan ampliamente las vertientes, por lo que la retención de suelos y escorrentía y la captación de agua de lluvia queda garantizada con la conservación y cuidado de los mismos.

Su elevada calidad visual se debe a la concurrencia de muchos factores. Suelen hallarse próximos a unidades de relieve singular (meandros, escarpes, hoyetas, etc), en los que existen frecuentes afloramientos rocosos. Además, la vegetación existente en la unidad presenta importantes contrastes cromáticos, tanto si están cultivadas (variedad de especies cultivadas) como si no lo están (recolonización vegetal pluriespecífica). 
Existen muy buenos ejemplos de procesos de recolonización natural en bancales en los diferentes ambientes geoecológicos de la cuenca baja y media del Guiniguada.

El paisaje circundante suele incrementar el valor paisajístico de la unidad de bancales, bien por tratarse de espacios escasamente urbanizados y con una calidad edificatoria media o alta, o por encontrase estos bancales enmarcados por espacios densamente vegetados con especies de elevado valor ecológico. En este sentido, existen enclaves con rodales de acebuches (Olea europaea), palmeras (Pboenix canariensis), etc. A todo lo expuesto, se suma la presencia de infraestructura hidráulica y agrícola, en muchas ocasiones, aunque en estados de conservación muy desigual y tipologías muy variadas, con un alto valor patrimonial.

\section{Discusión y conclusiones}

A lo largo de las páginas precedentes se ha presentado el diseño metodológico utilizado para la valoración de la calidad para la conservación de unidades de bancales en un sector de la cuenca del Guiniguada de Gran Canaria. El procedimiento concluye con la elaboración de un algoritmo que toma, como valores determinantes para la conservación, la calidad productiva, ecológica y estética de las unidades de bancales.

Aplicado el algoritmo, los resultados obtenidos ponen de manifiesto que el paisaje de bancales de la cuenca media y baja del Guiniguada presenta una calidad para la conservación situada en niveles medios y altos. El $71 \%$ de las unidades detectadas se sitúan en alguna de estas dos categorías, aunque sólo el $23 \%$ se incluyen en la categoría de alta calidad. Sin embargo se trata de una cifra importante, especialmente si se tiene en cuenta que las transformaciones socioeconómicas producidas en la segunda mitad del siglo XX se manifestaron de forma intensa en el abandono de los cultivos en bancales y condujeron a un importante deterioro de los mismos. En general, puede afirmarse que las prácticas agrícolas tradicionales han originado un paisaje cultural de gran calidad estética, que se superpone al heterogéneo y ya de por sí bello natural preexistente (cañón, meandros, rampas lávicas, conos volcánicos).

Desde el punto de vista metodológico, señalamos la adaptación del concepto "unidad de paisaje" al estudio de los bancales. Mediante la determinación de unidades que engloban campos de cultivo de similares características, la utilización de este concepto ha permitido una ágil interpretación y comparación del espacio estudiado, en el sentido de que facilita el inventario, la cartografía y la obtención de los diagnósticos.

Por otro lado, el algoritmo obtenido parece ajustarse bastante bien a la realidad de la cuenca, al tiempo que integra variables descriptivas y de diagnóstico parciales 
dispares. Es ahí precisamente donde radica la principal potencialidad de la utilización de los diagnósticos territoriales, especialmente de los de calidad para la conservación, al permitir una valoración acorde con los valores y limitaciones del espacio considerado e independiente de la procedencia académica de los técnicos encargados de la valoración, que requiere sólo de las oportunas adaptaciones territoriales. En esta línea, cada vez se hace más necesaria la investigación en procedimientos de diagnóstico que faciliten la toma de decisiones sobre un territorio y una temática considerada.

Sin embargo, y a pesar de la eficacia mostrada por el algoritmo empleado en este trabajo, se han detectado una serie de cuestiones que son susceptibles de futuras mejoras. Entre ellas se encuentra la carga de subjetividad implícita en algunos de los parámetros empleados, especialmente en los que contribuyen a la valoración de la calidad visual, así como la posibilidad de incluir más variables que pueden contribuir a explicar el valor patrimonial de los bancales, como pueden ser la existencia de otros elementos constructivos (contrafuertes, dobles muros, etc.) o de sistemas de regularización de la escorrentía.

Finalmente, uno de los aspectos en los que habrá que insistir en el futuro es la mejora del procedimiento de agregación definitivo, de forma que puedan establecerse, al menos, dos categorías más en la valoración final, lo que a su vez obliga a desarrollar un proceso previo de afinado de las categorías de valoración parcial.

\section{Bibliografía}

Ambroise, R., Frapa, P., Giorgis, S. (1989): Paisajes de terrasses, Aix-en-Provence. Edisud.

Bovet, T., Ribas, J. (1992): Metodología general de los estudios de paisaje. En BOLÓS, M. (dir.): Manual de Ciencia del Paisaje. Ed. Masson, Barcelona, p. 123-134.

Colomar Marí, A. (dir.) (2002): Patrimoni de marjades a la mediterrània occidental. Una proposta de catalogació. Consell de Mallorca, Departament de Promoció y Ocupació. FODESMA. Palma de Mallorca.
García Ruiz, J.M., Lasanta, T., Sobrón, I. (1988): Problemas de evolución geomorfológica en campos abandonados: el valle del Jubera (Sistema Ibérico). Zubia, 6, p. 99-114.

Lasanta, T. (1989): Evolución reciente de la agricultura de montaña: el Pirineo aragonés. Logroño, Geoforma Ediciones.

Lasanta, T. (1990): L'agriculture en terrasses dans les Pyrénées centrales espagnoles. Méditerranée, 71 (3-4), p. 37-42.

Llorens, P., Latron, J., Gallart, F. (1992): Analysis of the role of agricultural abandoned 
terraces of the hydrology and sediment dynamics in a small mountainous basin (High Llobregat, Eastern Pyrenees). Pirineos, 139, p. 27-46.

Marzol, V. (1987): El régimen anual de las lluvias en el Archipiélago Canario, Eria, 12, p. 187-194.

Pérez Cueva, A.J. (1985): Geomorfologia del sector ibérico valenciano entre los ríos Mijares y Turia. Tesis doctoral, Universidad de Valencia, $653 \mathrm{pp}$.

Rodríguez Aizpeolea, J. (1990): Evolució i situació actual dels bancals abandonats en el Parc natural del Montgó. Aiguaits, 5, p. $19-54$.

Rodríguez Aizpeolea, J., Lasanta Martínez, T. (1992): Los bancales en la agricultura de la montaña mediterránea: una revisión bibliográfica. Pirineos, 139, p. 105-123.

Romero, L.E., Hernández, L. (1996): Características pluviométricas de la cuenca del Guiniguada (1950-1994). Gran Canaria, Islas Canarias. En Marzol et al. (eds.): Clima y agua: la gestión de un recurso climático, La Laguna, p. 155-169.

Romero, L.E., Ruiz-Flaño, P., Hernández, L. (2000): Estudio y proposición de actuacio- nes para la conservación y restauración de las terrazas agricolas en el ámbito territorial del Proyecto Guiniguada (Gran Canaria, Islas Canarias). Proyecto encargado por el Cabildo Insular de Gran Canaria (Informe).

Sánchez, J., Ríos, C., Pérez-Chacón, E., Suárez, C. (1995): Cartografía del potencial del medio natural de Gran Canaria. Cabildo Insular de Gran Canaria, Las Palmas de Gran Canaria.

Spencer, J.E., Hale, G.A. (1961): The origin nature and distribution of agricultural terracing. Pacific Viewpoint, 2 (1), p. 1-40.

Tavio Álvarez, F., Ruiz Flaño, P., Hernández Calvento, L., Pérez-Chacón Espino, E. (2002): Propuesta metodológica para la elaboración de diagnósticos de calidad para la conservación: aplicación a la Vega de Guatiza-Mala (Lanzarote). En Longares Aladrén y Peña Monné, J.L. (eds.): Aportaciones geográficas en memoria del profesor L. Miguel Yetano Ruiz. Publicaciones del Departamento de Geografía y Ordenación del Territorio de la Facultad de Filosofía y Letras de la Universidad de Zaragoza, p. 519-527, Zaragoza.

Wheatley, P. (1965): Agricultural terracing. Pacific Viewpoint, 6, p. 123-144. 